\title{
Quantification of Left Atrial Tissue Remodeling Using Intra-Atrial Dyssynchrony by Cardiac Magnetic Resonance Imaging
}

\author{
Patrick T. Bering ${ }^{1}$ e João L. Cavalcante 2,3 \\ Medstar Washington Hospital Center, ${ }^{1}$ Washington - District of Columbia \\ Minneapolis Heart Institute - Abbott Northwestern Hospital, ${ }^{2}$ Minneapolis - Minnesota \\ Valve Science Center - Minneapolis Heart Institute Foundation, ${ }^{3}$ Minneapolis - Minnesota \\ Short Editorial related to the article: Intra-Atrial Dyssynchrony Using Cardiac Magnetic Resonance to Quantify Tissue Remodeling in Patients \\ with Atrial Fibrillation
}

Morphological and functional characteristics of the left atrium (LA) play a key role in the pathogenesis of atrial fibrillation (AF), which represents a global health burden as the most common cardiac arrhythmia encountered in clinical practice. ${ }^{1}$ For patients with drug-refractory $A F$, catheter-ablation may aid in a) prolonged restoration of sinus rhythm, b) decreased in total arrhythmic burden, symptomatic improvement, and c) better quality of life. However, catheter-ablation may not have a durable effect for a significant number of patients despite repeated procedures. ${ }^{2}$ A variety of innovative procedural technologies aim to improve patient freedom from AF. Scientific progress in the identification of patient characteristics that suggest a favorable or poor likelihood of procedural success may enhance patient selection for catheter-ablation and optimize time utilization for the cardiac electrophysiologist.

Cardiac magnetic resonance $(\mathrm{CMR})$ imaging with late gadolinium enhancement (LGE) has been shown to be a promising, non-invasive tool for the measurement of LA fibrosis, which predicts the recurrence of AF after catheter-ablation. ${ }^{3,4}$ While this tissue characterization of the LA represents a promising technology for patients with $A F$ in whom catheter-ablation is being considered, it remains mostly these days at expert centers, has labor-intensive post-processing and necessitates the use of gadolinium contrast, which may exclude patients who have advanced kidney disease or allergic reactions to gadolinium. Functional assessment with intra-atrial dyssynchrony utilizing tissue-tracking represents

\section{Keywords}

Atrial Fibrillation; Remodeling Atrial; Atrial Dyssynchrony Diagnostic Imaging; Magnetic Resonance Imaging.

Mailing Address: João L. Cavalcante •

Director, Cardiac MRI, Structural Cardiovascular Imaging and Core Labs Minneapolis Heart Institute - 800 East $28^{\text {th }}$ Street, Minneapolis, MN, 55407 Email: joao.cavalcante@allina.com

DOI: $10.5935 / a b c .20190073$ an elegant technique to characterize LA mechanics that does not require gadolinium contrast or significant post-processing and was recently shown to correlate with the recurrence of AF after catheter-ablation. ${ }^{4}$

In this issue, Ciuffo et al. ${ }^{5}$ advance the understanding of adverse LA remodeling and dysfunction in patients with AF. Using CMR to measure intra-atrial dyssynchrony in sinus rhythm, defined as the standard deviation of the time to the peak longitudinal strain [SD-TPS (\%)] and pre-atrial contraction strain [SD-TPS ${ }_{\text {preA }}(\%)$ ] corrected by the cycle length. LA fibrosis was quantified using LGE images, which, interestingly, did not differ significantly between paroxysmal and persistent AF types. Notably, SD-TPS was significantly higher in patients with persistent $\mathrm{AF}$ than those with paroxysmal $\mathrm{AF}$, although this association did not hold true for SD-TPS ${ }_{\text {preA }}$ between the AF types. On multivariable adjustment for age, sex, BMI, AF type, history of heart failure, OSA, hypertension, minimal LA volume and maximum LA longitudinal strain, both SD-TPS and SD-TPS remained significantly associated with LA LGE, although the signal was much stronger for SD-TPS. Inter- and intra-reader reproducibility was excellent for both indices, and the data were post-processed in a short amount of time.

These findings highlight the potential for intra-atrial dyssynchrony by CMR to represent a fast and accurate surrogate of LA fibrosis, especially in the prediction of AF recurrence after catheter-ablation. ${ }^{3,4}$ The authors have appropriately acknowledged the potential for selection bias in their non-randomized, retrospective cohort, and this technique requires patients to be in sinus rhythm at the time of CMR imaging. Still, Ciuffo et al. ${ }^{5}$ have added valuable insights into the understanding of LA remodeling in AF and must be praised for their work, which studied a real-world population and considered the important concern of work-flow for CMR post-processing. Their findings should stimulate more research into the use of intra-atrial dyssynchrony as non-invasive risk stratification for patients with $\mathrm{AF}$, which does not require gadolinium contrast to enhance patient selection for invasive therapies such as catheter-ablation. 


\section{References}

1. Chugh SS, Havmoeller R, Narayanan K, Singh D, Rienstra M, Benjamin EJ, et al. Worldwide epidemiology of atrial fibrillation: a Global Burden of Disease 2010 Study. Circulation. 2014;129(8):837-47.; 129(8): 837-847.

2. Calkins H, Hindricks G, Cappato R, Kim Y-H, Saad EB, Aguinaga L, et al. 2017 HRS/EHRA/ECAS/APHRS/SOLAECE expert consensus statement on catheter and surgical ablation of atrial fibrillation. Heart Rhythm. 2017;14(10):e275-444.

3. Marrouche NF, Wilber D, Hindricks G, Jais P, Akoum N, Machlinski F, et al Association of Atrial Tissue Fibrosis Identified by Delayed Enhancement
MRI and Atrial Fibrillation Catheter Ablation: The DECAAF Study. JAMA. 2014;311(5):498-506.

4. Ciuffo LA, Tao S, Gucuk Ipek E, Zghaib T, Balouch M, et al. Intra-Atria Dyssynchrony During Sinus Rhythm Predicts Recurrence After the First Catheter Ablation for Atrial Fibrillation. JACC Cardiovasc Imaging. 2019;12(2):310-9.

5. Ciuffo LA, Lima J, de Vasconcellos HD, Balouch M, Tao S, Nazarian S, et al. Intra-Atrial Dyssynchrony Using Cardiac Magnetic Resonance to Quantify Tissue Remodeling in Patients with Atrial Fibrillation. Arq Bras Cardiol. 2019; 112(4):441-450. 